\title{
Effects of mixed training and overtraining on recoveries from amnesias in rats with visual cortical ablations
}

\author{
THACKERY S. GRAY and DONALD R. MEYER \\ The Ohio State University, Columbus, Ohio 43212
}

\begin{abstract}
This investigation was concerned with the question of whether amnesias for simple visual tasks which result from injuries to the visual cortex can be corrected via training on other visual tasks. Such effects were noted, but only when the problems shared a common mode of solution. It was also observed, in contradiction to a classical conclusion of Lashley, that extensive preoperative overtraining conveys some protection of postoperative performance of the blackwhite discrimination problem.
\end{abstract}

Many years ago, Chow (1952) trained monkeys on visual discrimination problems of kinds that are not ordinarily rememberable by animals with injuries to the temporal isocortex. After operation, he trained his subjects on three new discrimination problems, and then found that their performance of the tasks they had learned prior to surgery was impaired hardly at all. Therefore he concluded that the temporal-lobe "deficit is not a simple loss of memory traces, but rather the result of the specific habits being temporarily suppressed."

The statement has proved to be prophetic. Indeed, at this juncture, there is no proof whatever that the engrams of memories of any type of habit can be destroyed by injuries to any subsector of the cortex (Meyer \& Meyer, in press). Until very recently, the principle support for the theory that the cortex is involved in memory storage was that presented by Lashley (1935). He observed that rats that are prepared with injuries to the visual neocortex forget a preoperatively learned brightness problem, even though they can relearn the problem. However, it has now been demonstrated that his subjects were suffering from amnesias, that is, from impairments of access to memories whose central neural substrates were intact (Meyer, 1972; cf. Meyer \& Meyer, 1977, for review).

At present, the latter conclusion is supported by two different kinds of observations. The first is that even though performance of the task is completely

This research was supported by Grant MH-06211 from the National Institute of Mental Health to Donald R. Meyer and Patricia M. Meyer. The authors extend their thanks to Mark Cloud and Robert Dewberry for their valuable technical assistance and to P. M. Meyer for her counsel with respect to the design of the investigation. Requests for reprints should be addressed to the Laboratory of Comparative and Physiological Psychology, Kinnear Research Center, The Ohio State University, 1314 Kinnear Road, Columbus, Ohio 43212. and permanently suppressed by ablations of the posterior sector of the cortex, treatments with amphetamine, which have no effect upon initial rates of learning of the task, will nonetheless accelerate postoperative relearning of the task (Braun, Meyer, \& Meyer, 1966; Jonason, Lauber, Robbins, Meyer, \& Meyer, 1970). The second is that even though untreated preparations will not ordinarily exhibit any signs of having learned the task prior to surgery, the engrams established by preoperative practice will thereafter strongly interfere with the learning of postoperative reversals of the task (LeVere \& Davis, 1977; LeVere \& Morlock, 1973). Such results are uninterpretable in terms of the concept that memories for the problem are stored at the level of the cortex; instead, they offer powerful support for the thesis that storage is accomplished by subcortical systems regardless of whether the animal is normal or not.

In this investigation, we have asked if the amnesias caused by posterior injuries are treatable with methods that do not involve explicit retraining on the task to be remembered. The approach was conceptually similar to Chow's (1952) in that we assessed the performance of problems that posterior subjects had learned prior to surgery as a function of their having had postoperative training or retraining on another kind of problem. Thus, we were searching for what we shall describe as crossed inductions of retrieval. The empirical question was whether crossed training would yield facilitation of relearning, and whether the amounts would be greater than can be accounted for in terms of postoperative transfer of training between the two problems.

\section{GENERAL METHODS}

\section{Subjects}

The subjects were 110 Long-Evans male hooded rats that were 90-120 days of age when they were given their initial training. The animals were housed in individual cages and maintained on an ad- 
lib food and water schedule. There were 11 groups with 10 subjects each, which were studied in three experiments.

\section{Apparatus}

The subjects were trained and/or retrained in a ThompsonBryant (1955) two-choice discrimination apparatus. The apparatus had three main components: a startbox, a choice compartment, and a goalbox. The walls of the apparatus were constructed of matte-finished black Plexiglas. The startbox and choice compartment were separated by a transparent guillotine door that was lifted by the experimenter at the beginning of each training trial. A partition between the choice compartment and the goalbox contained two doorways which were $9 \times 9 \mathrm{~cm}$ square and $7.6 \mathrm{~cm}$ apart. The interiors of the startbox and the choice compartment were illuminated by a group of small fluorescent lamps that were attached to transparent Plexiglas lids. The goalbox also had a transparent lid, but was not directly illuminated.

\section{Pretraining and Training Procedures}

The animals were pretrained and trained using the methods described by Glendenning (1972). First, each rat was handled for at least 5 min per day for a total of 5 days. Then, on Day 6, each rat was permitted to explore the apparatus for $15 \mathrm{~min}$. Next, on Day 7, the guillotine door was inserted between the startbox and the choice compartment, and the animal was trained to escape mild shocks which were delivered through a grid that formed the floors of the startbox and the choice compartment. Then pretraining doors were inserted into the doorways which led into the goalbox, and the subject was permitted to enter the goalbox after knocking down either of the doors.

On Day 8, the pretraining doors were replaced with training doors. Three different pairs of training doors were employed at one time or another. These pairs included: (1) a white door vs. a black door, (2) a large-checks door vs. a small-checks door, and (3) a door with left-oblique black-and-white stripes vs. a door with right-oblique black-and-white stripes. The stimuli are shown in Figure 1. The tasks presented with them will hereafter be described as the black-white $(\mathrm{BW})$, checks $(\mathrm{CH})$, and oblique-stripes $(\mathrm{OB})$ discrimination problems.

The luminances of the black and white components of the various cues were approximately 3 and $.1 \mathrm{~mL}$, respectively. The large $\mathrm{CH}$ door was composed of $16(4 \times 4)$ alternating black and white squares, while the small $\mathrm{CH}$ door was composed of 100 $(10 \times 10)$ alternating black and white squares. The black and white stripes of the $O B$ patterns were $1.27 \mathrm{~cm}$ wide. The ratio of amounts of contour in the $\mathrm{CH}$ patterns was 6.25 to 1 . When any of the problems were presented, the left-right positions of the stimuli were varied on a trial-to-trial basis according to a Gellerman

\section{CHECKS}

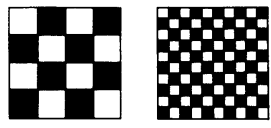

BLACK-WHITE

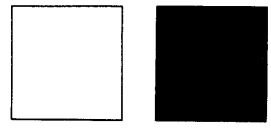

OBLIQUE-STRIPES

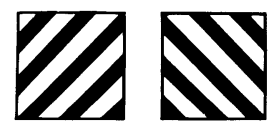

Figure 1. Stimulus pairs for the $\mathrm{BW}, \mathrm{CH}$, and $\mathrm{OB}$ problems.
(1933) series. When the $\mathrm{CH}$ and $\mathrm{OB}$ problems were presented, the doors were rotated on a trial-to-trial basis and according to another Gellermann series so that, in the long run, black or white checks or stripes were equally likely to be presented in the various subregions of the stimulus displays. The latter procedures were employed to suppress solutions of the $\mathrm{OB}$ and $\mathrm{CH}$ problems in terms of local-flux differentials, and thus to enforce solutions of the problems in terms of detections of edge orientations and amounts of differential contour, respectively.

When the problems were presented, the positive door was unlocked and the negative door was locked with a concealed mechanism. The positive cues were the white door, the large-checks door, and the left-oblique door. Training was conducted for 25 trials per day, with rests of approximately $10 \mathrm{~min}$ following the 8 th and the 16 th trials. The amounts of training on particular tasks were varied so that each of the subjects of a group would reach a criterion of 9 of 10 correct responses prior to termination of training on a task, and also so that every subject would receive the same amounts of training prior to surgery. Thus, for example, subjects that were trained on a CHBW combination were given 75 trials of training on the $\mathrm{CH}$ problem and then were trained for 25 trials on the BW problem. Subjects that were trained on a BWCH combination were given 50 trials of training on the BW problem and then were trained for 50 further trials on the $\mathrm{CH}$ problem. Hence total training was equated, but adjustments were made in the components of the training because the $\mathrm{CH}$ problem was substantially harder than the BW problem for subjects that had not been previously trained on the $\mathrm{CH}$ problem.

Similar adjustments of amounts of training were required when the animals were retrained after operation. At that stage, it also proved to be impossible to maintain a 100 -trial limitation and yet have all of the subjects of the groups rereach a criterion of $9 / 10$ on both of the postoperative tasks. Thus, one group that received BWCH training before operation relearned the BW task in 50 trials or less, and then relearned the $\mathrm{CH}$ problem in 50 trials or less. Another group, with comparable preoperative training, which was first retrained on the $\mathrm{CH}$ problem had to be retrained on the $\mathrm{CH}$ problem for 100 trials, but then, in every instance, rereached criterion on the BW problem after 25 further trials or less. Also, subjects with no preoperative experience had to be trained on the $\mathrm{CH}$ problem for 125 trials.

The preoperative training for some of the subjects was concentrated on a single task. The groups are designated as OBOT, CHOT, and BWOT. Under those conditions, the animals were trained on the $\mathrm{OB}, \mathrm{CH}$, and $\mathrm{BW}$ problems, and then were given overtraining on their tasks until they had each received a total of 4 days and 100 trials of training. After operation, the subjects of the three groups were always first retrained on the BW problem and then on the $\mathrm{CH}$ problem, and thus they were given $50 \mathrm{BW}$ trials followed by $50 \mathrm{CH}$ trials. But all of the subjects of all of the groups, regardless of their differential treatments, were tested for retention and for transfer of training after having received exactly the same amounts of training prior to operation.

\section{Surgical Procedures}

The surgeries were performed with clean technique while the rats were under anesthesias induced by sodium pentobarbital. The lesions were intended to extend from the sagittal sinus to the rhinal sulci, and from the bregma backward to the occipital pole. The ablations were produced by the pia-stripping method in conjunction with aspiration at the margins of the injuries (cf. Meyer \& Meyer, 1971). In Experiments 1 and 2, the posterior cortex was destroyed in one stage. In Experiment 3, a serial procedure was employed in which the left and then the right posterior cortex, or vice versa, were destroyed in two stages. The subjects with onestage bilateral ablations were given 12 days to recover. The subjects with two-stage serial ablations were given 12 days to recover from both their first- and second-stage ablations. The animals were also given prophylactic treatments with a broad-band antibiotic following the surgeries. 


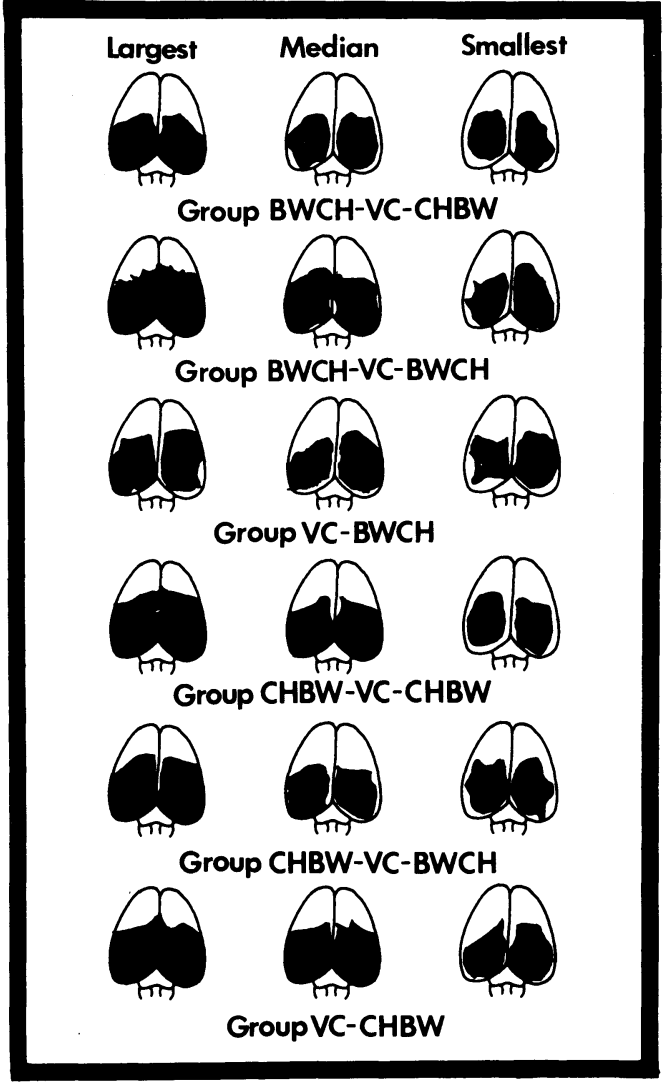

Figure 2. Histological results for Experiment 1.

\section{Histological Procedures}

After the experiments were finished, the subjects were given lethal injections of sodium pentobarbital and were then perfused with normal saline followed by $10 \%$ Formalin. The brains were removed and the extents of the cortical ablations were charted on Lashley diagrams. The findings were essentially the same as those reported for approximately 1,000 posterior subjects that have previously been studied on our laboratory's program with respect to inductions of recoveries (cf. Meyer \& Meyer, 1977). Hence, in Figure 2, we show the smallest, largest, and median injuries to the posterior cortex of the 60 subjects that were studied in Experiment 1 . The other 50 subjects had ablations of comparable extents. None of the injuries resulted in serious invasions of subcortical structures.

\section{EXPERIMENT 1}

The first experiment was designed to explore the relationship between a normal animal's performances of $\mathrm{CH}$ and $\mathrm{BW}$ problems, and to ask if those relationships would be altered as a function of bilateral injuries to the visual cortex. There were six groups of subjects, of which four were trained in various orders on the tasks before and after the rats had sustained one-stage complete bilateral visual cortical (CVC) ablations. The first four groups were designated CHBWCVC-CHBW, CHBW-CVC-BWCH, BWCH-CVC$\mathrm{BWCH}$, and BWCH-CVC-CHBW. To illustrate, Group CHBW-CVC-CHBW learned the checks problem and then the black-white problem prior to surgery. After operation, the subjects were retrained on the checks and then on the black-white problem. The other two groups were not preoperatively trained on either of the tasks, and hence were designated CVC-BWCH and CVC-CHBW.

Table 1 presents the means and standard errors of the means for the numbers of trials that the six groups of subjects required to meet the $9 / 10$ criterion during each phase of preoperative and/or postoperative training. The outstanding findings were as follows. The groups first trained on the BW problem learned that task in approximately 27 trials, while those first trained on the $\mathrm{CH}$ problem learned that task in approximately $\mathbf{4 0}$ trials. Both kinds of training yielded positive preoperative transfer. Thus, preoperative BW training reduced the trials required for $\mathrm{CH}$ learning to approximately 24 trials, and initial preoperative $\mathrm{CH}$ training enabled the subjects to learn the $\mathrm{BW}$ problem in about 5 trials. After operation, the subjects that were first retrained on the $\mathrm{BW}$ problem took about 21 retraining trials to learn the problem, and those which were first retrained on the $\mathrm{CH}$ problem took approximately 55 retraining trials to learn the problem. Postoperative transfer was also observed in that the subjects which were first retrained on the $\mathrm{CH}$ problem (Groups BWCH-CVC-CHBW and CHBW-CVC-CHBW) afterwards relearned the BW problem in about 4 trials, and those which were first retrained on the BW problem (Groups BWCHCVC-BWCH and CHBW-CVC-BWCH) afterwards relearned the $\mathrm{CH}$ problem in approximately 16 trials.

The subjects with preoperative BWCH or CHBW training were quicker to relearn the $\mathrm{BW}$ problem or the $\mathrm{CH}$ problem as a first postoperative task than were the subjects which had not been trained on either task prior to surgery. Thus, Group CVC$\mathrm{CHBW}$ took about 65 trials to learn the $\mathrm{CH}$ problem, as contrasted with the transfer groups' scores of 53 and 57. Group CVC-BWCH took 29 trials to learn the $\mathrm{BW}$ problem, as contrasted with the transfer groups' scores of 23 and 20 . But the differences, as tested with Mann-Whitney Us, were not statistically significant.

Table 1

Means and Standard Errors of the Means for Groups of Subjects in Experiment 1

\begin{tabular}{|c|c|c|c|c|c|c|c|c|}
\hline \multirow[b]{3}{*}{ Group } & \multicolumn{4}{|c|}{ Preoperative } & \multicolumn{4}{|c|}{ Postoperative } \\
\hline & \multicolumn{2}{|c|}{ Task 1} & \multicolumn{2}{|c|}{ Task 2} & \multicolumn{2}{|c|}{ Task 1} & \multicolumn{2}{|c|}{ Task 2} \\
\hline & Mean & SE & Mean & SE & Mean & SE & Mean & SE \\
\hline BWCH-CVC-CHBW & 26.9 & 1.9 & 25.9 & 3.3 & 53.2 & 5.8 & 3.3 & .7 \\
\hline CHBW-CVC-CHBW & 41.8 & 4.3 & 5.3 & 1.4 & 56.7 & 5.5 & 4.4 & 1.5 \\
\hline CVC-CHBW & & & & & 64.9 & 7.2 & 5.8 & 1.4 \\
\hline BWCH-CVC-BWCH & 27.4 & 2.2 & 22.7 & 2.7 & 22.5 & 3.8 & 17.4 & 2.3 \\
\hline CHBW-CVC-BWCH & 39.6 & 2.9 & 5.5 & 1.2 & 20.3 & 3.1 & 15.3 & 3.5 \\
\hline CVC-BWCH & & & & & 28.5 & 2.1 & 41.9 & 4.1 \\
\hline
\end{tabular}


The most intriguing feature of the outcomes was that neither type of preoperative training yielded a substantial amount of initial postoperative protection of performance of either the $\mathrm{BW}$ or the $\mathrm{CH}$ problem. Thus, for example, the BW score of about 23 for Group BWCH-CVC-BWCH was not very different from what would be expected had the subjects not been given preoperative $\mathrm{CH}$ training. Indeed, Glendenning's (1972) score for what would be described as a Group BW-CVC-BW was 22.8 trials, although, in his experiment, the subjects received approximately a quarter of the number of preoperative trials that were given to Group BWCH-CVC-BWCH.

In summary, then, Experiment 1 indicated that mixed-task training prior to surgery does not protect postoperative performance of the BW problem, that mixed-task training effects are independent of the orders in which the tasks are presented prior to surgery, that training on either of the tasks facilitates performance of the other task, and that injuries to the cortex will not prevent the transfer from occurring. Measurements were also obtained for the rates of $\mathrm{CH}$ learning and relearning and of the amounts of transfer which are noted if normal or operated subjects are given $\mathrm{CH}$ training following $\mathrm{BW}$ training. And, finally, the outcomes suggested that a subject with $\mathrm{CH}$ training has also learned the $\mathrm{BW}$ problem, but that $\mathrm{CH}$ performances are not as powerfully affected by BW training.

\section{EXPERIMENT 2}

In the second study, the question was whether postoperative training on the BW problem would yield a task-specific facilitation of performance of a problem that had been overlearned prior to surgery. Three groups of animals were studied. The subjects were trained and then overtrained on the BW problem, the $\mathrm{CH}$ problem, or the $\mathrm{OB}$ problem. The groups were designated as BWOT-CVC-BWCH, CHOTCVC-BWCH, and OBOT-CVC-BWCH. The animals were given 100 trials of preoperative training on the problems. Then they were prepared with bilateral posterior ablations. After a rest of 12 days, they were given 50 trials of practice with the $\mathrm{BW}$ problem. Thereafter, the subjects were given 50 trials of practice with the $\mathrm{CH}$ problem, which proved to be sufficient for all of the animals to reach the criterion of $9 / 10$ on that task.

Table 2 presents the results. The first important finding was that BWOT yielded a substantial protection of postoperative performance of the $\mathrm{BW}$ problem. The second was that $\mathrm{OB}$ overtraining conveyed no detectable protection of postoperative performance of the BW problem. There was also a suggestion that CHOT affected the BW score. However, the only statistically significant effect in the Task 1
Table 2

Means and Standard Errors of the Means for Groups of Subjects in Experiment 2

\begin{tabular}{|c|c|c|c|c|c|c|}
\hline \multirow[b]{3}{*}{ Group } & \multirow{2}{*}{\multicolumn{2}{|c|}{$\begin{array}{c}\text { Preoperative } \\
\text { Learning } \\
\end{array}$}} & \multicolumn{4}{|c|}{ Postoperative Performance } \\
\hline & & & \multicolumn{2}{|c|}{ Task 1} & \multicolumn{2}{|c|}{ Task 2} \\
\hline & Mean & SE & Mean & SE & Mean & SE \\
\hline BWOT-CVC-BWCH & 22.0 & 1.9 & 13.9 & 2.0 & 15.9 & 1.9 \\
\hline CHOT-CVC-BWCH & 38.7 & 1.9 & 19.4 & 2.8 & 19.4 & 3.0 \\
\hline OBOT-CVC-BWCH & 40.5 & 2.3 & 24.7 & 2.2 & 27.6 & 2.8 \\
\hline
\end{tabular}

scores was the difference between the BWOT and OBOT outcomes.

The Task 2 results for $\mathrm{CH}$ learning or relearning showed that preoperative BWOT had much the same effect upon performance of the problem as preoperative CHOT. The difference between the scores of 16 and 19 , respectively, was not significant, as tested with the Mann-Whitney method. Also, and importantly, the scores were very close to the Task 2 postoperative scores of Groups BWCH-CVC-BWCH and CHBW-CVC-BWCH, which were studied in Experiment 1 (approximately 17 and 15 trials, respectively). The OBOT Task 2 score was substantially higher than the others, but consistently less than the Task 2 score of $\mathbf{4 2}$ trials for Group CVC-BWCH, which was studied in Experiment 1.

In summary, then, the findings of Experiment 2 showed that extensive BWOT protects postoperative performance of the BW problem, that protection of retention of the BW problem is not effected by comparable amounts of OBOT, and that BW training after operation has much the same effect upon postoperative $\mathrm{CH}$ performances of subjects which were given either BWOT or CHOT prior to surgery. Hence, although Task 1 postoperative training resulted in an evident facilitation of Task 2 performance of the $\mathrm{CH}$ problem by the BWOT and CHOT subjects, the results for the two groups indicated that preoperative training on the $\mathrm{CH}$ problem conveyed no particular advantage to the CHOT subjects.

\section{EXPERIMENT 3}

Experiment 3 was prompted by the finding that OBOT, although it failed to yield a demonstrable effect upon postoperative learning of the BW problem, appeared to have a modest effect upon the rate at which the Task $2 \mathrm{CH}$ problem was learned by the subjects of Group OBOT-CVC-BWCH. The finding was interesting because the $\mathrm{OB}$ problem is completely and permanently insoluble by rats with injuries to the posterior cortex (Horel, Bettinger, Royce, \& Meyer, 1966; Jonason et al., 1970; Lavond, Hata, Gray, Geckler, Meyer, \& Meyer, 1978). Hence, if there is, in fact, a consequence of OB training for postoperative performance of the $\mathrm{BW}$ problem, the conse- 
Table 3

Means and Standard Errors of the Means for Groups of Subjects in Experiment 3

\begin{tabular}{|c|c|c|c|c|c|c|}
\hline \multirow[b]{2}{*}{ Group } & \multicolumn{2}{|c|}{$\begin{array}{c}\text { Pre- } \\
\text { operative }\end{array}$} & \multicolumn{2}{|c|}{$\begin{array}{c}\text { Inter- } \\
\text { operative }\end{array}$} & \multicolumn{2}{|c|}{$\begin{array}{c}\text { Post- } \\
\text { operative }\end{array}$} \\
\hline & Mean & SE & Mean & SE & Mean & SE \\
\hline $\begin{array}{l}\text { BW-UVC-BW-UVC-BW } \\
\text { BW-UVC-BR-UVC-BW }\end{array}$ & 24.6 & 2.9 & $\begin{array}{r}3.5 \\
605\end{array}$ & $\begin{array}{r}.8 \\
55\end{array}$ & $\begin{array}{r}6.3 \\
173\end{array}$ & 1.6 \\
\hline
\end{tabular}

quence is uninterpretable in terms of transfer of training that is mediated via reactions to discriminative cues that are present in both situations.

Two groups of subjects were studied. Both groups were given preoperative BW training and overtraining. The day after training, the subjects were prepared with unilateral posterior (UVC) ablations. After recovery from their first-stage surgeries and beginning on the 12th day thereafter, one group was given another 100 trials on the BW problem. The other group was given 100 trials of training on the OB problem. When interoperative training was completed, that is, 4 days later, both groups of subjects were prepared with second-stage ablations of the contralateral posterior cortex. Following recovery from the second-stage surgeries, the animals were retrained on the BW problem. Hence, the first group was designated as Group BWOT-UVC-BWOT-UVC$\mathrm{BW}$. The second group was designated as Group BWOT-UVC-OBOT-UVC-BW.

The results are summarized in Table 3. It presents the means and standard errors of the means for the preoperative, interoperative, and postoperative trials that were required for learning or relearning to the 9/10 criterion. The preoperative scores were essen- tially the same as those for the comparable groups of subjects in Experiments 1 and 2. Group BWOT-UVCBWOT-UVC-BW rapidly relearned the problem following the first-stage ablations; their score of 3.5 mean trials was the lowest that has been observed in our program for subjects which are retrained on the problem following a unilateral posterior injury. The subjects which were given OBOT between operations required about as many trials to learn the task as naive normal animals, and about twice as many as unilateral subjects with preoperative $O B$ training (Lavond et al., 1978).

Both groups of subjects relearned the BW problem following their second operations in substantially fewer trials than are required for subjects which are trained to criterion on the problem, are prepared with one-stage posterior ablations, and then are retrained on the problem (cf. Meyer \& Meyer, 1977). Accordingly, the fact that the group which was trained on the OB problem between successive surgeries were able to relearn the task in 17 trials, instead of about 25 , suggested that a serial effect can be obtained through interoperative training on a task that is unsolvable by subjects with complete posterior ablations. However, we will show that the conclusions must be guarded because the latter group was also given preoperative BWOT, before we realized that extensive preoperative practice with the task conveys protection.

\section{DISCUSSION}

The present observations are relatable to outcomes obtained in several prior investigations. Hence, in Table 4, we summarize results from experiments con-

Table 4

Synopsis of Results of the Present and Related Studies

\begin{tabular}{|c|c|c|c|c|c|c|c|c|c|}
\hline Number & Study & $\mathbf{N}$ & PRE-1 & PRE-2 & SURG-1 & IOTR & SURG-2 & POST-1 & POST-2 \\
\hline 1 & HBRM* & 33 & & & CVC & & & BW29 & \\
\hline 2 & HBRM* & 33 & BW25 & & CVC & & & BW26 & \\
\hline 3 & MYUM* & 23 & BW27 & & CVC & & & BW24 & \\
\hline 4 & MYDM* & 41 & BW26 & & CVC & & & BW24 & \\
\hline 5 & KBMM* & 10 & BW24 & & CVC & & & BW24 & \\
\hline 6 & GLEN & 14 & BW25 & & CVC & & & BW23 & \\
\hline 7 & GLEN & 15. & BW27 & BWOT & CVC & & & BW27 & \\
\hline 8 & KBMM* & 79 & BW23 & & UVC & & UVC & BW22 & \\
\hline-9 & GLEN & 16 & BW25 & & UVC & BWOT & UVC & BW13 & \\
\hline 10 & ALEE & 14 & BW25 & & UVC & BWOT & UVC & BW07 & \\
\hline 11 & LHGM & 17 & OB52 & & UVC & OB32 & UVC & OBFL & BW29 \\
\hline 12 & GRAY & 10 & & & CVC & & & BW29 & $\mathrm{CH} 42$ \\
\hline 13 & GRAY & 10 & OB41 & OBOT & CVC & & & BW25 & $\mathrm{CH} 28$ \\
\hline 14 & GRAY & 10 & CH39 & СHOT & CVC & & & BW19 & CH19 \\
\hline 15 & GRAY & 10 & BW22 & BWOT & CVC & & & BW14 & $\mathrm{CH} 16$ \\
\hline 16 & GRAY & 10 & BW27 & $\mathrm{CH} 24$ & CVC & & & BW23 & $\mathrm{CH} 17$ \\
\hline 17 & GRAY & 10 & CH40 & BW06 & CVC & & & BW20 & $\mathrm{CH} 15$ \\
\hline 18 & GRAY & 10 & & & CVC & & & CH65 & BW06 \\
\hline 19 & GRAY & 10 & BW27 & $\mathrm{CH} 26$ & CVC & & & CH53 & BW03 \\
\hline 20 & GRAY & 10 & $\mathrm{CH} 42$ & BW05 & CVC & & & CH57 & BW04 \\
\hline 21 & GRAY & 10 & BW25 & BWOT & UVC & BWOT & UVC & BW06 & \\
\hline 22 & GRAY & 10 & BW26 & BWOT & UVC & OBOT & UVC & BW17 & \\
\hline
\end{tabular}

*Studies in which the massed-trial procedure was employed and the scores have been divided by two. 
ducted with a total of $\mathbf{4 0 5}$ male Long-Evans rats. The following codes are employed to identify the sources: HBRM refers to Horel, Bettinger, Royce, \& Meyer (1966); MYUM to Meyer, Yutzey, and Meyer (1966); MYDM to Meyer, Yutzey, Dalby, and Meyer (1968); GLEN to Glendenning (1972); KBMM to Kircher, Braun, Meyer, and Meyer (1970); ALEE to an unpublished study of Aileen Lee that is described by Meyer and Meyer (1977); LHGM to Lavond, Hata, Gray, Geckler, Meyer, and Meyer (1978); and GRAY to the present report.

All of the data in the table were collected in experiments in which measures of rates of relearning of the BW problem by rats with injuries to the visual cortex were obtained. The injuries either were completed in one stage (CVC) or were serial unilateral (UVC-UVC) ablations. PRE-1 and PRE-2 describe the preoperative training. $\mathrm{BW}, \mathrm{OB}$, and $\mathrm{CH}$ refer to the black-white, oblique-stripes, and checks discrimination problems. BWOT, OBOT, and CHOT mean that the subjects were given overtraining after having met a criterion of $9 / 10$. IOTR describes the interoperative treatments of subjects that had been prepared with serial UVC ablations, and POST-1 and POST-2 describe postoperative training. The unusual entry OBFL means that the subjects failed to relearn the OB discrimination problem.

The numbers in the columns give the mean spacedtrial or estimated mean spaced-trial scores for learning or relearning of the various kinds of problems. In the older studies, which are marked with asterisks, the animals were trained with a massed-trial method that was first employed by Horel et al. (1966). The rest were conducted with the spaced-trial method of Glendenning (1972), which yields scores that are close to one-half the scores which are obtained when the massed-trial method is employed (cf. Glendenning, 1972; Howarth, Meyer, \& Meyer, 1979). Accordingly, the estimated spaced-trial entries were obtained by dividing the massed-trial mean scores by 2 .

Approximately 20 years elapsed between the times that the earliest and latest sets of data in the table were collected. However, the preoperatively naive subjects of Group 1-HBRM learned the BW problem in 58 massed trials, which yielded an accurate prediction that the subjects of Group 12-GRAY would learn the problem in 29 spaced trials. Also, in the older studies, in which the PRE-1 condition was learning of the BW problem, the means for the observed or estimated scores were $25,27,26,24,25,27$, 23,25 , and 25 (Groups 2-10), and in this investigation the spaced-trial scores for five groups of subjects which were similarly treated were $22,27,27,25$, and 26 (Groups 15, 16, 19, 21, and 22). Hence, we propose to cross-compare the quantitative findings which the table summarizes, regardless of whether they are from the present study or from previous studies in the program.

\section{Effects of Preoperative Overtraining}

The present study was concerned with the problems posed by the POST-1 findings for Groups 1-6. Why is it that a CVC ablation will completely suppress performance of a problem that the animal can nonetheless relearn? And what can be done to help the subject to remember the problem? The 7-GLEN experiment, which served to replicate a classical experiment of Lashley (1921), had suggested that preoperative overtraining conveys no protection whatsoever. The findings for 8-KBMM, which confirmed a result of Petrinovich and Carew (1969), had also indicated that it doesn't matter whether the injuries are inflicted in one or two stages. However, the 8-GLEN and 9-GLEN studies, which confirmed an observation of Thompson (1960), showed that protection of ultimate retention can be obtained provided that serial preparations are given retraining on the BW problem following their first-stage ablations.

In this investigation, we have found that extensive preoperative BW overtraining will, in fact, permit a CVC subject to relearn the problem with savings. It is likely that the failures of Glendenning (1972) and Lashley (1921) to observe the effect were due to the fact that their animals received considerably less overtraining than did the subjects of the 15-GRAY experiment. However, interoperative retraining on the problem conveys more protection than comparable amounts of preoperative overtraining. Thus, in 9-GLEN, subjects which received a total of 35 preoperative trials (i.e., 25 trials plus 10 criterial trials) and 31 IOTR trials exhibited about the same amount of savings as the subjects of the 15-GRAY study. Also, in 10-ALEE, in which the animals were given 41 trials of IOTR, the 76 total trials conveyed more protection than the 100 trials of preoperative training that was given to the 15-GRAY subjects.

The POST-1 results of 21-GRAY and 10-ALEE were virtually the same. Hence, there appears to be a limit as to how much protection of ultimate retention can be obtained through BW overtraining prior to and following a first-stage UVC ablation. Thus, in 10-ALEE, there was no preoperative overtraining, and her subjects were interoperatively retrained for less than half as many trials as the subjects of the 21-GRAY experiment.

Although there was a modest suggestion in 14GRAY that preoperative $\mathrm{CH}$ overtraining conveys some protection of performance of the $\mathrm{BW}$ problem, neither that procedure nor the OBOT that was given prior to surgery in the 13-GRAY study yielded a significant result. Moreover, interoperative OBOT, when combined in the 22-GRAY experiment with preoperative BWOT, had no detectable effect whatsoever upon the POST-1 BW scores of the subjects. The latter group relearned the BW problem faster than the 8-KBMM subjects, but the difference appeared to be attributable to the fact that the 8-KBMM 
group received no preoperative BW overtraining. Thus, if anything, the POST-1 scores of the subjects of the 22-GRAY experiment were somewhat inferior to those of the subjects of the 15-GRAY investigation, which also were preoperatively overtrained and were tested for retention of the BW problem after having been prepared with one-stage CVC ablations.

Here we will observe that in the 22-GRAY, but not in the 11-LHGM and 13-GRAY investigations, the animals were trained on the BW problem prior to being given overtraining on the $\mathrm{OB}$ problem. Hence, although the findings of the latter two studies had already shown that OBOT does not affect the rate of initial acquisitions of the BW problem by UVC-UVC or CVC preparations, there still remained a question of whether the procedure would serve to enhance the recallability of a previously established memory. A positive answer would have offered strong support for the concept of crossed induction, but neither original learning nor relearning of the $\mathrm{BW}$ problem by posterior subjects appears to be significantly affected by either preoperative or interoperative $\mathrm{OB}$ training.

\section{Effects of Mixed Preoperative Training}

CVC subjects which are given extensive BW overtraining prior to surgery relearn the problem with a savings of about 10 spaced trials (cf. POST-1 15GRAY with POST-1 2-HBRM, 3-MYUM, 4-MYDM, 5-KBMM, and 6-GLEN). The savings are not just a function of overall amounts of preoperative training, as is clear from the OBOT results and also from the POST -1 consequences of mixed preoperative $\mathrm{CH}$ and $\mathrm{BW}$ training. Thus, the $\mathrm{BWCH}$ subjects and $\mathrm{CHBW}$ subjects which were studied in the 16- and 17-GRAY investigations required about 23 and 20 spaced trials, respectively, to relearn the $\mathrm{BW}$ problem following their CVC ablations. Those scores are evidently close to expectations for CVC subjects that are given BW training but are not overtrained, and indicate that no particular advantage is conveyed by "broad" preoperative training insofar as postoperative remembering of the BW problem is concerned.

However, at first glance, the POST-2 results of the 16- and 17-GRAY experiments suggest that postoperative BW training yields a crossed induction of postoperative remembering of the $\mathrm{CH}$ problem by preoperatively mixed-trained subjects. Thus, in 12GRAY, CVC subjects required about 42 trials to learn the problem after having first been trained during POST-1 on the BW problem. In contrast, the POST-2 $\mathrm{CH}$ scores for the mixed-trained subjects were 17 and 15 spaced trials, or about one-half as many trials as were required by the subjects of the 12-GRAY study. Also, those scores were considerably lower than the POST-2 score for $\mathrm{CH}$ learning by the subjects of Group 13-GRAY.

However, comparisons between the POST-2 scores of Groups 14- through 17-GRAY serve to destroy the argument. The difficulty is that subjects that were given preoperative $\mathrm{CH}$ overtraining and then were trained on the BW problem during POST-1 learned the $\mathrm{CH}$ problem at about the same rate as subjects that were given mixed preoperative training or preoperative BW overtraining. Hence, although postoperative $\mathrm{BW}$ training reduces the trials required for CVC subjects to relearn the checks discrimination, it is clear from the overall results that such effects are completely independent of whether or not the animals had previously formed preoperative memories for the $\mathrm{CH}$ problem.

\section{The Common Characteristics of the $\mathrm{CH}$ and BW Problems}

We shall next be concerned with the question of why the POST-2 performances of mixed-trained subjects were excellent despite the fact that such performances could not be attributed to crossed inductions of retrieval of preoperative memories. We have, thus far, ignored the outcomes obtained for CVC subjects that were given mixed training and then were first retrained on the $\mathrm{CH}$ problem rather than the BW problem. However, just as POST-1 BW training facilitates performance of the $\mathrm{CH}$ problem during POST-2, POST-1 training on the $\mathrm{CH}$ problem facilitates performance of the BW problem during POST-2 (Groups 19- and 20-GRAY). Also, a naive CVC subject, if first given training on the $\mathrm{CH}$ problem prior to being trained on the BW problem after surgery, will learn the latter problem in a very small fraction of the customary number of trials (cf. POST-2 of 18-GRAY with POST-1 of 12-GRAY and 1-HBRM).

Normal rats, if trained on either of the problems, also exhibit considerable amounts of transfer when trained on the other problem during PRE-2 (cf. the PRE-1 results of studies $2-10,16,19,21$, and 22 with the PRE-2 findings of 17- and 20-GRAY, and the PRE-1 findings of 14-GRAY and 17-GRAY with the PRE-2 findings of 16- and 19-GRAY). The largest effects are obtained when the subjects are first given $\mathrm{CH}$ training, which reduces the trials required for BW learning from approximately 25 to 6 . Hence, all the data from the mixed-trained subjects suggest that the $\mathrm{CH}$ and $\mathrm{BW}$ problems share a common mode of solution, and that the common mode is probably employed to a greater extent during $\mathrm{CH}$ training than it is during $\mathrm{BW}$ training.

We suspect that the transfers are functions of the fact that the BW problem, as typically presented, is not just a "flux" or "brightness" discrimination problem. Thus a black door upon a black background is both a dim and edgeless stimulus, while a white door upon a black background is both a bright and edged stimulus. The edged vs. edgeless difference is a cue that normal rats respond to when trained on the BW problem, as is shown by the findings of an unpublished study in our laboratory by G. D. Ritchie 
and Timothy Field. The observations were that if the black and white doors are displayed upon a neutral gray background, a procedure which serves to eliminate the edge differential, normal rats require about 32 spaced trials instead of 25 to learn the problem.

The $\mathrm{CH}$ problem, as presented in this study, is a task that is insoluble in terms of either overall or local differences in flux. Hence, when a subject is trained on the problem after having learned the BW problem, the animal cannot employ the habit that it learned in relation to the BW flux differential. However, the habit that it learned in relation to the $\mathrm{BW}$ edge differential is transferable because the $\mathrm{CH}$ problem can also be solved as a differential contour problem. But one would not expect a particularly striking amount of transfer on those grounds, for the findings of Ritchie and Field indicate that edge-related BW habits are weaker than flux-related BW habits.

From such considerations, we believe that a rat which is first given training on the $\mathrm{CH}$ problem learns, among other things, a habit of responding to differential-contour cues. Hence, if the animal is subsequently trained on the BW problem, it already has a basis of solution of the latter problem which is supplemented by the addition of a flux differential. The concept accounts for the fact that such a subject will quickly learn the BW problem quickly, while a rat that is trained on the $\mathrm{CH}$ problem is helped to some extent and one that is trained on the BW problem is helped to a lesser extent.

There are, of course, some other conceivable bases for transfer of training on the $\mathrm{CH}$ problem to the $\mathrm{BW}$ problem, and vice versa. However, inasmuch as posterior injuries will not prevent the transfer from occurring, an interpretation of the transfer in terms of a habit that is learnable in both situations requires that the postulated habit be within the competence of CVC subjects. The requirement is satisfied by habits that are learned on a differential-contour basis (e.g., Mize, Wetzel, \& Thompson, 1971), but at present there is no proof that CVC subjects can learn to discriminate between a pair of cues that have been very carefully equated with respect to amounts of visual flux and visual contour (cf. Ritchie, Meyer, \& Meyer, 1976 , or Meyer \& Meyer, in press, for review).

\section{Conclusions}

Although the experiments described in this report were necessarily complex, our principal conclusions from the data are comparatively simple. First, overtraining on the black-white problem induces a protection of postoperative performance of the task by posterior preparations. That result qualifies the previous conclusions of Lashley (1921) and, more recently, Glendenning (1972). However, inasmuch as the protection thus obtained is demonstrably weaker than that which is conveyed by equivalent amounts of overtraining of a subject with a preexisting poste- rior injury, the results still permit us to believe in the validity of Thompson's (1960) serial effect.

Second, we encountered no convincing suggestion that postoperative performances of simple visual habits are salubriously affected by mixed or "broad" preoperative training. Nor did we observe that mixedtrained subjects, if given retraining on one of the problems after surgery, are thereby enabled to remember the other task as well. Accordingly, our findings offer no support for the concept of crossed induction, that is, for generalization of the findings of Chow (1952) with respect to temporal monkeys. Here we will observe that such a generalization has been proposed by Meyer and Meyer (in press), and that this investigation was designed with the aim of giving it empirical foundation.

We doubt that our failure to observe crossed induction was due to our having used inadequately sensitive procedures. Thus, although postoperative transfer of training on the $\mathrm{CH}$ problem to the $\mathrm{BW}$ problem was so great as to render that particular paradigm unsuitable for tests of the concept, the POST-2 $\mathrm{CH}$ performances of subjects that were first retrained on the BW problem were poor enough to suggest that there was room for improvement of performance via crossed induction of retrieval. We continue to be puzzled by one of the results, namely, that preoperative OBOT appears to facilitate relearning of the $\mathrm{CH}$ problem by rats that have had postoperative training on the $\mathrm{BW}$ problem. We believe that the finding is significant enough to warrant a further exploration. However, we will stress that it offers no support for the concept of crossed induction because there are no common bases of solution of the $\mathrm{OB}$ and $\mathrm{CH}$ problems.

In closing, we will note that the program of which the present studies were a part has been aimed for many years at the discovery of bases for improvements in therapies for strokes. A part of that battle has already been won through our proofs that amnesias can be deep and enduring without necessarily implying that injuries which produce them destroy memorial substrates. But we still have a problem of understanding how those latent resources can be tapped, hopefully with methods that will not involve explicit retraining on the tasks to be remembered. Hence, our present findings are somewhat disappointing, except for those concerning overtraining, for their message seems to be that explicit retraining is a sine qua non for inductions of recoveries from the kinds of amnesias we have studied. Perhaps further studies will revise that conclusion; if so, we will be the first to cheer.

\section{REFERENCES}

Braun, J. J., Meyer, P. M., \& Meyer, D. R. Sparing of a brightness habit in rats following visual decortication. Journal 
of Comparative and Physiological Psychology, 1965, 61, 79-82.

CHow, K. L. Conditions influencing the recovery of visual discriminative habits in monkeys following temporal neocortical ablations. Journal of Comparative and Physiological Psychology, $1952,45,430-437$.

Gellermann, L. W. Chance orders of alternating stimuli in visual discrimination experiments. Journal of Genetic Psychology, 1933, 42, 207-208.

GLEnDENNING, R. L. Effects of training between two unilateral lesions of visual cortex upon ultimate retention of black-white habits by rats. Journal of Comparative and Physiological Psychology, 1972, 80, 216-229.

Horel, J. A., Bettinger, L. A., Royce, G. J., \& Meyer, D. R. Role of neocortex in the learning and relearning of two visual habits by the rat. Journal of Comparative and Physiological Psychology, 1966, 61, 66-78.

Howarth, H., Meyer, D. R., \& Meyer, P. M. Perinatal injuries to the visual cortex enhance the significance of extravisual cortex for performance of a visual habit. Physiological Psychology, 1979, 7, 163-166.

Jonason, K. R., Lauber, S. M., Robbins, M. J., Meyer, P. M., \& MEYER, D. R. Effects of amphetamine upon relearning pattern and black-white discriminations following neocortical lesions in rats. Journal of Comparative and Physiological Psychology, 1970, 73, 47-55.

Kircher, K. A., Braun, J. J., Meyer, D. R., \& Meyer, P. M. Equivalence of simultaneous and successive neocortical ablations in production of impairments of retention of black-white habits in rats. Journal of Comparative and Physiological Psychology, $1970,71,420-425$.

Lavond, D. G., Hata, M. G., Gray, T. S., Geckler, C. L., MEYer, P. M., \& MEYER, D. R. Visual form perception is a function of the visual cortex. Physiological Psychology, 1978, 6, 471-477.

LASHLEY, K. S. Studies of cerebral function in learning. II. The effects of long-continued practice upon cerebral localization. Journal of Comparative Psychology, 1921, 1, 453-468.

LASHLEY, K. S. The mechanism of vision: XII. Nervous structures concerned in habits based on reactions to light. Comparative Psychology Monographs, 1935, 11, 43-79.

LEVERE, T. E., \& DAVIS, N. Recovery of function after brain damage: The motivational specificity of spared neural traces. Experimental Neurology, 1977, 57, 883-889.
LEVere, T. E., \& Morlock, G. W. Nature of visual recovery following posterior neodecortication in the hooded rat. Journal of Comparative and Physiological Psychology, 1973, 68, 62-67.

Meyer, D. R. Access to engrams. American Psychologist, 1972, 27, 124-133.

MeYer, D. R., \& MEYer, P. M. Dynamics and bases of recoveries of functions after injuries to the cerebral cortex. Physiological Psychology, 1977, 5, 133-165.

MEYer, D. R., \& MEYeR, P. M. Inductions of recoveries from amnesias. In J. L. McGaugh \& R. F. Thompson (Eds.), Learning and memory: Handbook of behavioral neurobiology. New York: Plenum Press, in press.

Meyer, D. R., Yutzey, D. A., \& Meyer, P. M. Effects of neocortical ablations on relearning of a black-white discrimination habit by two strains of rats. Journal of Comparative and Physiological Psychology, 1966, 61, 83-86.

MeYer, P. M., \& MEYER, D. R. Neurosurgical procedures with special reference to aspiration lesions. In R. B. Myers (Ed.), Methods in neurobiology. New York: Academic Press, 1971.

Meyer, P. M., Yutzey, D. A., Dalby, D. A., \& Meyer, D. R. Effects of simultaneous septal-visual, septal-anterior and anteriorposterior lesions upon relearning a black-white discrimination. Brain Research, 1968, 8, 281-290.

Mize, R. R., Wetzel, A. B., \& Thompson, V. E. Contour discrimination in the rat following removal of posterior neocortex. Physiology \& Behavior, 1971, 6, 861-867.

Petrinovich, L., \& Carew, T. J. Interaction of neocortical lesion size and interoperative experience in retention of a learned brightness discrimination. Journal of Comparative and Physiological Psychology, 1966, 61, 136-138.

Ritchie, G. D., Meyer, P. M., \& Meyer, D. R. Residual spatial vision of cats with lesions of the visual cortex. Experimental Neurology, 1976, 53, 227-254.

Thompson, R. Retention of a brightness discrimination following neocortical damage in the rat. Journal of Comparative and Physiological Psychology, 1960, 53, 212-215.

Thompson, R., \& BRYANT, J. R. Memory as affected by activity of the relevant receptor. Psychological Reports, 1955, 1, 393-400.

(Received for publication June 30, 1980; accepted October 27, 1980.) 